\title{
Uso de atracúrio para o bloqueio da musculatura extrínseca do bulbo ocular em cães submetidos à anestesia inalatória sob ventilação espontânea
}

\author{
[Blocking of ocular bulb extrinsic musculature by atracurium in inhalant anesthetized dogs \\ under spontaneous breathing] \\ A.B. Carregaro ${ }^{1}$, P.M. Gerardi ${ }^{2}$, M.C.L.C. Mujalli ${ }^{2}$, F.S. Martins $^{2}$ \\ ${ }^{1}$ Departamento de Clínica de Pequenos Animais - UFSM \\ Faixa Nova de Camobi, km 9, número 1000 \\ 97105-900 - Santa Maria, RS \\ ${ }^{2}$ Médico veterinário autônomo
}

\begin{abstract}
RESUMO
Comparou-se o bloqueio da musculatura extrínseca do bulbo ocular com três doses de atracúrio em cães submetidos à anestesia inalatória sob ventilação espontânea. Em estudo cego, foram utilizados seis cães, pré-medicados com $0,1 \mathrm{mg} / \mathrm{kg}$ de acepromazina intravenoso (IV), anestesiados com $5 \mathrm{mg} / \mathrm{kg}$ de propofol, entubados e mantidos sob anestesia inalatória com $1,5 \%$ de isofluorano e submetidos a quatro tratamentos: não tratados (controle), tratados com $25 \mu \mathrm{g} / \mathrm{kg}(\mathrm{G} 25)$ de atracúrio IV, com 50 $\mathrm{gg} / \mathrm{kg}$ (G50) de atracúrio IV e com $75 \mu \mathrm{g} / \mathrm{kg}(\mathrm{G} 75)$ de atracúrio IV. Mensuraram-se: pressão parcial de $\mathrm{CO}_{2}$ expirado $\left(\mathrm{ETCO}_{2}\right)$, freqüência cardíaca $(\mathrm{FC})$, freqüência respiratória $(f)$, saturação arterial de oxiemoglobina $\left(\mathrm{SatO}_{2}\right)$ e tempo de centralização do bulbo ocular. Nos grupos tratados com atracúrio, o $\mathrm{ETCO}_{2}$ aumentou aos 5min, e permaneceu aumentado até $10 \mathrm{~min}$ em G50 e até $20 \mathrm{~min}$ em G75, sendo este o único tratamento cuja concentração de $\mathrm{ETCO}_{2}$ apresentou-se acima de $50 \mathrm{mmHg}$. Não houve reinalação de $\mathrm{CO}_{2}$ em nenhum grupo. Em G75, observou-se aumento crescente da $f$ até os 40min e considerável bradicardia após 10min; ambos retornaram aos valores basais após esse período. A centralização do bulbo ocular foi

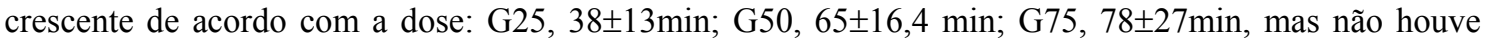
diferença estatística entre G50 e G75. Conclui-se que G50 apresentou bloqueio satisfatório sem promover intensa e prolongada hipercapnia nos animais.
\end{abstract}

Palavras-chave: cão, atracúrio, hipercapnia

\begin{abstract}
The ocular bulb extrinsic musculature blocking by the administration of three atracurium doses in isoflurane anesthetized dogs under spontaneous breathing was compared. In a blind study, six dogs were premedicated with $0.1 \mathrm{mg} / \mathrm{kg}$ of acepromazine, anesthetized with $5 \mathrm{mg} / \mathrm{kg}$ of propofol, intubated and maintained in inhalation anesthesia with $1.5 \%$ of isoflurane in 100\% of oxygen. Afterwards, they were submitted to four treatments

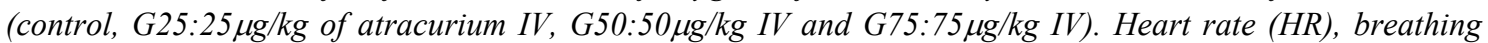
rate (BR), $\mathrm{CO}_{2}$ extrated (ETCO2), arterial saturation of oxyhaemoglobin ( $\left.\mathrm{SatO}_{2}\right)$ and ocular bulb centralization time were measured. $\mathrm{ETCO}_{2}$ in all animals were increased at 5min keeping high until 10min in G50 dogs and until 20min in G75 dogs; this was the unique animal group that showed results above $50 \mathrm{mmHg}$ of $\mathrm{ETCO}_{2} . \mathrm{CO}_{2}$ was not reinhaled by any animal. It was observed an increase in BR until 40min and a considerable bradycardia after 10min in G75 animals; both returned to basal levels thereon. The

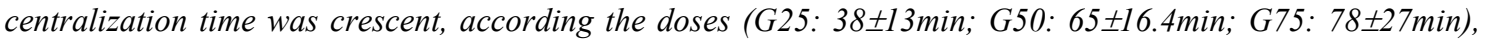
but no difference between G50 and G75 animals was observed. It was showed that G50 dogs took a content ocular centralization without intense and prolonged hypercapnia.
\end{abstract}

Keywords: dog, atracurium, hypercapnia

Recebido em 29 de março de 2005

Aceito em 18 de agosto de 2006

E-mail: carregaro@smail.ufsm.br 


\section{INTRODUÇÃO}

Os miorrelaxantes periféricos são comumente utilizados na anestesiologia veterinária como adjuvantes para a realização de intervenções oftálmicas, torácicas, ortopédicas ou decorrentes de alterações na freqüência e amplitude respiratórias, facilitando, assim, as manipulações cirúrgicas (Boldy, 1988; LeCorre et al., 1999; McManus, 2001).

Nunes e Laus (1995), em revisão de literatura sobre técnicas anestésicas em cirurgias oculares, citaram que, para o bom andamento das cirurgias intraoculares, há necessidade da centralização do bulbo ocular, em que a córnea encontrar-se-á totalmente visível. Para isso, pode-se optar pelo uso de bloqueadores neuromusculares a fim de promover um relaxamento da musculatura circunvizinha ao bulbo ocular.

A atuação dos miorrelaxantes periféricos é caracterizada por paralisia flácida obtida em velocidades diferentes, de acordo com as regiões corpóreas. Geralmente os músculos crâniocervicais são acometidos primeiro, seguidos pelos da cauda, membros, deglutição e laringe. Os abdominais, intercostais e o diafragma são os últimos a seguirem a seqüência de paralisação. Em resumo, há um bloqueio progressivo, da periferia ao centro. A recuperação se dá de forma inversa (Muir et al., 2001).

Apesar de existirem duas classes distintas de miorrelaxantes periféricos, os despolarizantes e não-despolarizantes, a utilização da primeira tem sido minimizada em virtude dos efeitos adversos, como miosites, devido à despolarização tetanizante e estimulação do sistema cardiovascular, culminando em arritmias, hipercalemia e inibição da metabolização hepática (Ostergaard et al., 1989; Markewitz e Elstad, 1997; Nagujb e Magboul, 1998).

A utilização dos miorrelaxantes nãodespolarizantes na rotina anestésica tem aumentado consideravelmente devido à ausência de efeito tetanizante e facilidade de biotransformação (Jakhirani et al., 1990; Rang et al., 2001). O pancurônio, pertencente a esse grupo, na dose de $0,06 \mathrm{mg} / \mathrm{kg}$ IV em cães, promove bloqueio muscular de longa duração, resultando em apnéia devido à paralisia do diafragma (Spruell, 1980). Lee et al. (1998) analisaram a depressão respiratória e miorrelaxamento em cães tratados com pancurônio, concluindo que doses entre 0,01 a $0,02 \mathrm{mg} / \mathrm{kg}$ IV proporcionaram centralização do bulbo ocular e moderada depressão respiratória, sem, contudo, promoverem apnéia.

O atracúrio difere do pancurônio por possuir uma única via de degradação, sendo mais seguro para pacientes hepato e/ou nefropatas (Jakhrani et al., 1990). A molécula é instável e degrada-se facilmente por eliminação de Hoffmann, não utilizando a função hepática e renal para a biotransformação e excreção. Seus metabólitos são inativos como bloqueadores, porém a laudanosina pode produzir estimulação simpática e convulsões se em altas concentrações na corrente sangüínea (Vizi e Lendvai, 1997).

Em um procedimento cirúrgico, um dos parâmetros analisados para avaliar a eficiência ventilatória é a pressão parcial de $\mathrm{CO}_{2}$ expirado $\left(\mathrm{ETCO}_{2}\right)$ (Moon, 1990). Devido à extrema rapidez com que o $\mathrm{CO}_{2}$ se difunde entre os tecidos, há um certo equilíbrio entre o $\mathrm{CO}_{2}$ capilar $\left(\mathrm{PaCO}_{2}\right)$ e o alveolar, apesar de esse último ser ligeiramente menor que o anterior, porém desprezível sob o ponto de vista clínico (Teixeira-Neto et al., 2002). Haskins (1996) afirma que, em condições normais de ventilação, a $\mathrm{PaCO}_{2}$ oscila entre 35 e $45 \mathrm{mmHg}$. Para isso, a $\mathrm{PaCO}_{2}$ pode ser determinada por capnometria em pacientes submetidos à anestesia inalatória que não possuam marcantes alterações cardiopulmonares (Magi et al., 1994; Cheng et al., 1999).

O estudo objetivou promover o bloqueio da musculatura extrínseca do bulbo ocular, empregando-se o atracúrio por via IV, em cães submetidos à anestesia inalatória com isofluorano sob ventilação espontânea, e determinar a dose desse miorrelaxante sem ocasionar alterações ventilatórias.

\section{MATERIAL E MÉTODOS}

Para o estudo cego proposto, foram utilizados seis cães hígidos, machos ou fêmeas, sem raça definida e pesos entre 10 e $20 \mathrm{~kg}$. Todos foram autocontroles, ou seja, participaram de todos os grupos, anulando, assim, a variação individual. 
Com jejum alimentar de 12 horas e hídrico de quatro horas, os animais foram pré-medicados com $0,1 \mathrm{mg} / \mathrm{kg}$ de acepromazina ${ }^{1}$ intravenosa (IV). Após 15min, foram anestesiados com $5 \mathrm{mg} / \mathrm{kg}$ de propofol ${ }^{2}$, entubados com sonda orotraqueal de diâmetro adequado e mantidos sob anestesia inalatória com $1,5 \%$ de isofluorano ${ }^{3}$, diluído em $100 \%$ de $\mathrm{O}_{2}$, mantendose em segundo plano anestésico, caracterizado principalmente pela rotação do bulbo ocular e pelo reflexo palpebral.

Transcorrido o período de estabilização dos parâmetros fisiológicos (20min), os animais foram submetidos a quatro tratamentos, sendo um controle e três com bloqueio neuromuscular com $25 \mu \mathrm{g} / \mathrm{kg}$ de atracúrio ${ }^{4} \mathrm{IV}$ (G25), $50 \mu \mathrm{g} / \mathrm{kg}$ IV (G50) e $75 \mu \mathrm{g} / \mathrm{kg}$ IV (G75). O intervalo entre cada grupo foi de, no mínimo, sete dias.

O bloqueio da musculatura extrínseca do bulbo ocular foi avaliado pelo tempo em que o bulbo permanecia centralizado, de acordo com Nunes e Laus (1995). A freqüência cardíaca (FC) e o ritmo cardíaco foram avaliados através de monitor eletrocardiográfico ${ }^{5}$. A freqüência respiratória (FR), pressão parcial de $\mathrm{CO}_{2}$ expirado $\left(\mathrm{ETCO}_{2}\right)$ e inspirado e a saturação arterial de oxiemoglobina $\left(\mathrm{SatO}_{2}\right)$ foram mensuradas por oxicapinometria ${ }^{6}$.

Os parâmetros foram mensurados imediatamente antes da administração de atracúrio (tempo 0min) e aos $5,10,20,40,60,80$ e $100 \mathrm{~min}$. O tempo de centralização do bulbo ocular foi observado até o pronto restabelecimento daquela musculatura, quando o bulbo tornava-se rotacionado ou semirotacionado.

A análise estatística foi realizada, segundo Callegari-Jaques (2003), com o auxílio de programa estatístico ${ }^{7}$. Utilizou-se análise de repetição múltipla (ANAVA) para amostras relacionadas, para avaliação de diferenças dentro de cada grupo, comparando-se todos os momentos com o tempo 0min, seguido pelo teste de Dunnett, quando necessário. A comparação

\footnotetext{
${ }^{1}$ Acepran 0,2\%. Univet S.A., SP.

${ }^{2}$ Pronest. Meizler Com. Inter. S.A., SP.

${ }^{3}$ Isoforine. Cristália Prod. Farm. Ltda, SP.

${ }^{4}$ Tracur. Cristália Prod. Farm. Ltda, SP.

${ }^{5}$ Ecafix Active. Ecafix Ind. Com. Ltda, SP.

${ }^{6}$ DX7100 $\mathrm{CO}_{2}$ SMO. Dixtal Biom. Ind. Com Ltda, AM

${ }^{7}$ GraphPad Prism 4. GraphPad Software Inc.
}

entre os grupos foi realizada por ANAVA seguida pelo teste Tukey, quando necessário. Os valores foram expressos em média \pm erro-padrão e considerados significantes quando $\mathrm{P}<0,05$.

\section{RESULTADOS E DISCUSSÃO}

Todas as doses de atracúrio foram eficientes no bloqueio da musculatura extrínseca do bulbo ocular, promovendo a exposição total da córnea, condição adequada para intervenções intraoculares. Em G25, obteve-se um período de centralização de $38 \pm 13 \mathrm{~min}$, diferindo significantemente de G50 (65 $\pm 16,4 \mathrm{~min})$ e G75 (78 $\pm 27 \mathrm{~min})$. Não houve diferença significativa entre G50 e G75. Em G25, observou-se, ainda, a não centralização em um dos animais, permanecendo semi-rotacionado, por isso não foi considerado para efeito estatístico.

Os animais do grupo-controle permaneceram com o bulbo ocular rotacionado em todo o período mensurado, atestando a centralização exclusivamente ao miorrelaxante periférico. Salienta-se ainda a estabilização dos parâmetros analisados nesse grupo em todos os momentos, não diferindo em relação ao momento basal em nenhuma das variáveis.

Após o período de estabilização dos parâmetros fisiológicos (0min), todos os grupos apresentaram valores de $\mathrm{ETCO}_{2}$ similares, oscilando entre 33 e $36 \mathrm{mmHg}$. Cinco minutos após a administração de atracúrio, todos os animais apresentaram concentrações mais altas que as obtidas anteriormente. Todavia, comparadas às do grupo-controle, apenas em G50 e G75 as concentrações foram mais altas (Fig. 1).

Após $10 \mathrm{~min}$, o $\mathrm{ETCO}_{2}$ em $\mathrm{G} 25$ retornou próximo ao valor inicial $(38 \pm 9,7 \mathrm{mmHg})$, não mais diferiu em relação ao momento 0min, e assim permaneceu até o fïm das mensurações. Em G50, observou-se permanência do $\mathrm{ETCO}_{2}$ acima do inicial $(43 \pm 6,8 \mathrm{mmHg})$, diferindo também em relação ao grupo-controle. Entretanto, não houve diferença significativa em momento algum quando comparado ao G25. Após isso, houve decréscimo dessa variável, que não diferiu tanto em relação ao seu tempo 0min quanto ao controle. 


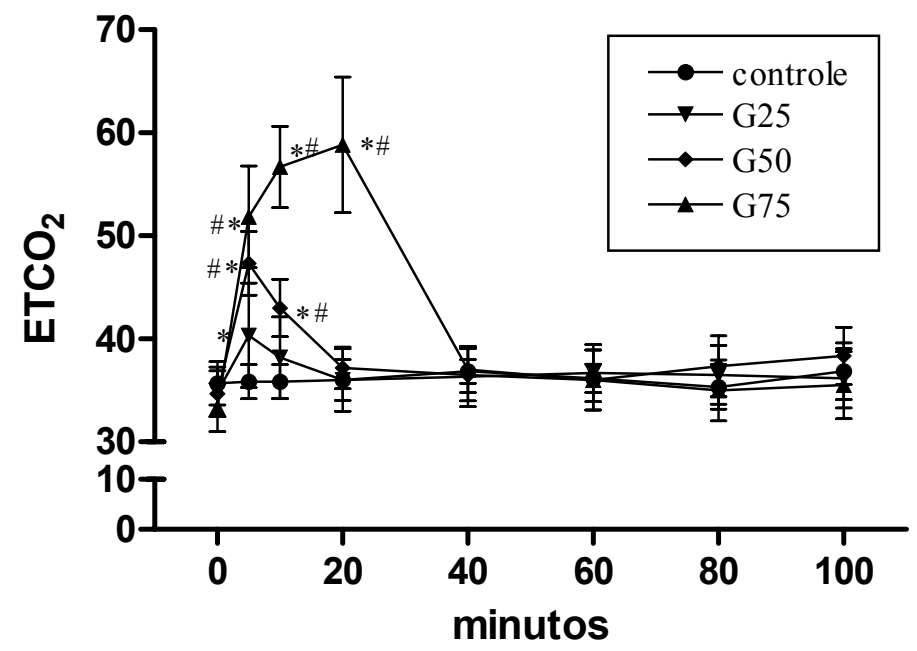

Figura 1. Pressões parciais de $\mathrm{CO}_{2}$ expirado $\left(\mathrm{ETCO}_{2}\right)$ obtidas em cães submetidos ao bloqueio da musculatura extrínseca do bulbo ocular pelo atracúrio IV. Valores expressos em média \pm erro-padrão.

* Diferença significante intragrupo em relação ao tempo $0 \min (\mathrm{P}<0,05)$.

\# Diferença significante intergrupo em relação ao controle $(\mathrm{P}<0,05)$.

Em G75, a concentração de $\mathrm{ETCO}_{2}$ permaneceu significativamente acima do valor inicial, dos 5 aos $20 \mathrm{~min}$, sempre superior a $50 \mathrm{mmHg}$. Comparada à dos demais grupos, essa foi mais elevada aos $10 \mathrm{e}$ $20 \mathrm{~min}$. Aos $40 \mathrm{~min}$, os valores obtidos em todos os grupos foram semelhantes, permanecendo assim até o fim das mensurações.

Apesar de, aos 5min, todos os grupos tratados com atracúrio apresentarem $\mathrm{ETCO}_{2}$ acima do basal, em G25, essa variável permaneceu dentro dos parâmetros fisiológicos. Em G50, apesar de não diferir estatisticamente do controle, verificaram-se valores acima de $45 \mathrm{mmHg}$, caracterizando hipercapnia. Todavia, já aos 10min, mesmo apresentando significância em relação ao basal, retornou aos valores permitidos, oscilando próximo a $37 \mathrm{mmHg}$ até o fim das mensurações. Nas concentrações obtidas em G75, constatou-se $\mathrm{ETCO}_{2}$ acima de $50 \mathrm{mmHg}$, que atingiu $59 \mathrm{mmHg}$ aos $20 \mathrm{~min}$, valores muito superiores aos propostos por Haskins (1996), demonstrando um quadro acentuado de hipercapnia. Grosenbaugh e Muir (1998), após testarem métodos não invasivos de monitoração, constataram que o $\mathrm{ETCO}_{2}$ subestima a $\mathrm{PaCO}_{2}$ quando os valores superam $55 \mathrm{mmHg}$ no aparelho, principalmente em pacientes hipotensos. Pode-se supor, desse modo, que a $\mathrm{PaCO}_{2}$ em G75, entre 10 e $20 \mathrm{~min}$, poderia ser superior ao $\mathrm{ETCO}_{2}$ mensurado.

A hipercapnia promovida leva à acidose respiratória, com a formação de ácido carbônico $\left(\mathrm{HCO}_{3}{ }^{-}\right)$, proveniente da reação entre $\mathrm{CO}_{2}$ e $\mathrm{H}_{2} \mathrm{O}$, podendo ser compensada mediante retenção de bicarbonato $\left(\mathrm{H}_{2} \mathrm{CO}_{3}\right)$ ou não, culminando em diminuição do pH (Luna, 2002). No presente estudo, essa decorreu da diminuição da força de contração da musculatura intercostal e do diafragma devido ao uso do bloqueador neuromuscular. Neste caso, houve diminuição da complacência pulmonar para realizar a fase de inspiração. Desse modo, os alvéolos não são completamente distendidos, aumentando o espaço morfofisiológico e dificultando a troca de gases (Reece, 1988).

Como era de se esperar, em G75, a FR aumentou aos $10 \mathrm{~min}$, embora esse aumento não tenha sido significativo em relação ao seu basal e aos outros grupos no mesmo momento. A elevação da FR nesse grupo foi gradual e, aos 40min, foi significantemente maior que nos demais grupos, decrescendo após isso (Fig. 2). Esse aumento deveu-se à diminuição da amplitude respiratória dos animais. Nesse caso, há aumento do $\mathrm{CO}_{2}$ sangüíneo que, por sua vez, atravessa a barreira hematoencefálica, dissociando-se com $\mathrm{H}_{2} \mathrm{O} . \mathrm{O} \mathrm{H}^{+}$ proveniente dessa reação exerce poderoso efeito estimulatório nos quimiorreceptores do bulbo cerebral (Guyton e Hall, 1997). Assim, houve compensação na manutenção do volume-minuto e da oxigenação. Tanto em G25 quanto em G50, não houve diferença significante em relação ao tempo 0 min e ao controle, o que pode demonstrar que, apesar do aumento do $\mathrm{ETCO}_{2}$ nesses grupos, não houve estimulação dos quimiorreceptores, talvez pelo rápido retorno dessa variável aos padrões fisiológicos. 


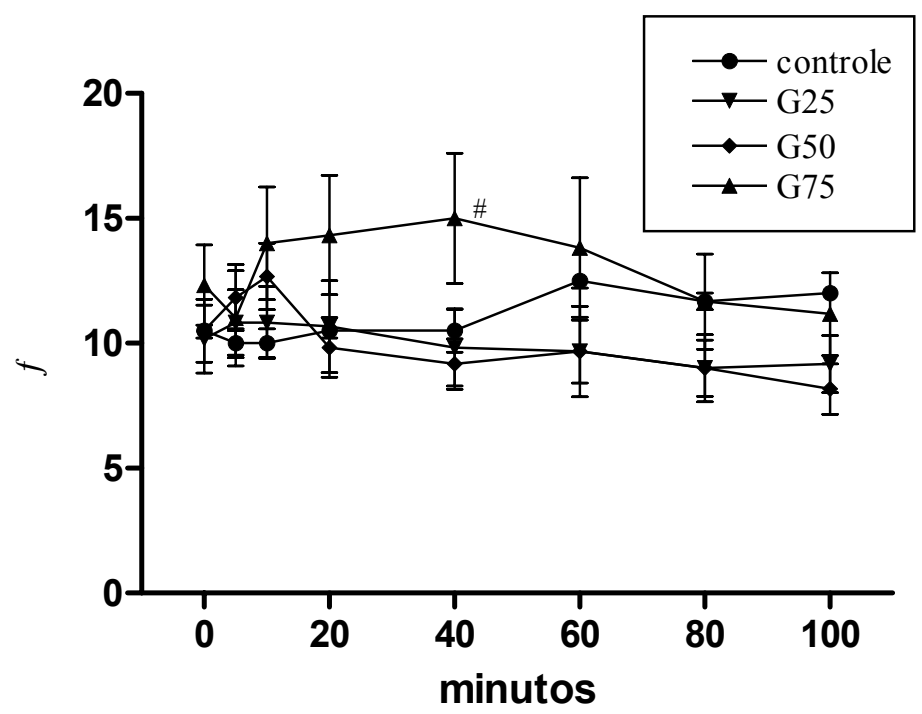

Figura 2. Freqüências respiratórias $(f)$ obtidas em cães submetidos ao bloqueio da musculatura extrínseca do bulbo ocular pelo atracúrio IV. Valores expressos em média \pm erro-padrão.

\# Diferença significante intergrupo em relação ao controle $(\mathrm{P}<0,05)$.

Vizi e Lendvai (1997) citaram que os bloqueadores neuromusculares promovem, em maior ou menor grau, taquicardia devido à ação antivagal. A taquicardia promovida pelo atracúrio somente é observada em infusões contínuas e basicamente é devido a seu metabólito, a laudanosina. Em G25 e G50, não houve alterações dignas de nota em relação à FC no grupo-controle. Em G75, houve um decréscimo aos $5 \mathrm{~min}$, sendo significante aos 10min, o que pode ser explicado, segundo Khanna et al. (1995), pela hipercapnia instalada (Fig. 3).

$\mathrm{O}$ aumento da $\mathrm{PaCO}_{2}$ promove estimulação simpática na tentativa de melhorar a oxigenação sangüínea. Todavia, quando ultrapassa $60 \mathrm{mmHg}$, há predomínio vagal, culminando em bradicardia e hipotensão. Apesar de a $\mathrm{PaCO}_{2}$ não ter sido mensurada nos animais, pela observação dos valores obtidos em G75 para $\mathrm{ETCO}_{2}$ e $\mathrm{FC}$, conclui-se que, nesse caso, o aumento de $\mathrm{CO}_{2}$ nos tecidos levou à bradicardia. Após o pronto reestabelecimento desse parâmetro, a FC retornou aos padrões fisiológicos normais. Não foi observada alteração no ritmo cardíaco em nenhum grupo.
A saturação arterial de oxiemoglobina por meio de mensuração não invasiva é relativamente precisa quando se encontra entre $70 \%$ e $100 \%$ (Grosenbaugh e Muir, 1998). Na $\mathrm{SatO}_{2}$, não se observou diferença significante entre grupos em relação ao respectivo valor basal e entre os grupos em nenhum momento. Todos os valores foram sempre acima de 97\% (Fig. 4). Com isso, atestou-se que, apesar do aumento do $\mathrm{ETCO}_{2} \mathrm{em}$ todos os grupos tratados com atracúrio, nenhum animal apresentou hipoxemia associada.

\section{CONCLUSÕES}

As três doses de atracúrio utilizadas neste estudo promoveram bloqueio da musculatura extrínseca do bulbo ocular sem paralisia completa das musculaturas intercostais e diafragmática. Os efeitos da dose de $50 \mu \mathrm{g} / \mathrm{kg}$ foram semelhantes ao da dose de $75 \mathrm{mg} / \mathrm{kg}$, sem promover alterações marcantes na fisiologia do paciente. Concluiu-se que a dose de $50 \mathrm{mg} / \mathrm{kg}$ de atracúrio é adequada para cirurgias oftálmicas que necessitem do bloqueio da musculatura extrínseca do bulbo ocular em animais submetidos à anestesia inalatória com isofluorano, sem a necessidade de ventilação controlada. 


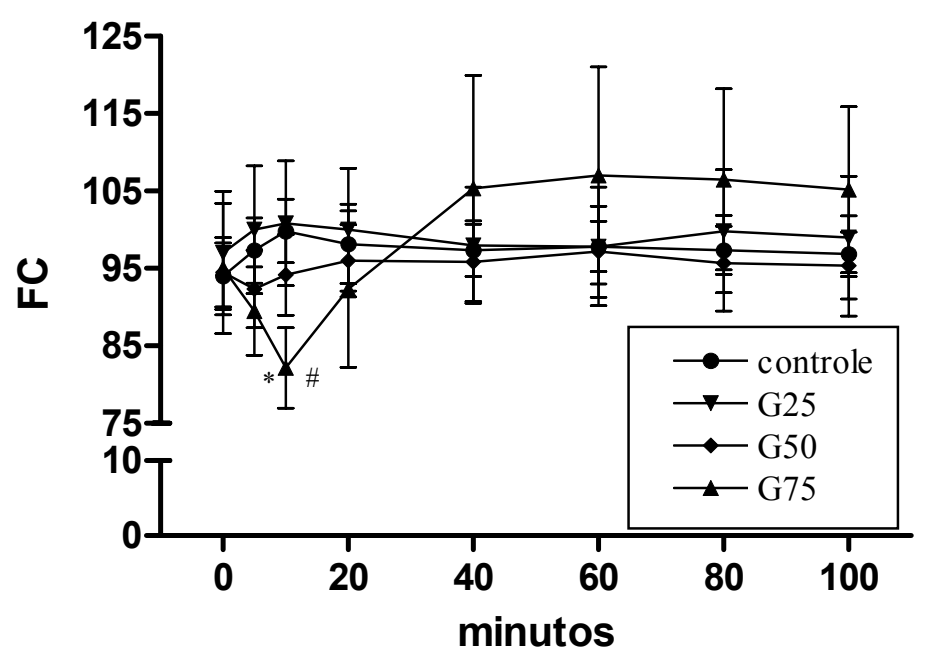

Figura 3. Freqüências cardíacas (FC) obtidas em cães submetidos ao bloqueio da musculatura extrínseca do bulbo ocular pelo atracúrio IV. Valores expressos em média \pm erro-padrão.

* Diferença significante intragrupo em relação ao tempo $0 \mathrm{~min}(\mathrm{P}<0,05)$.

\# Diferença significante intergrupo em relação ao controle $(\mathrm{P}<0,05)$.

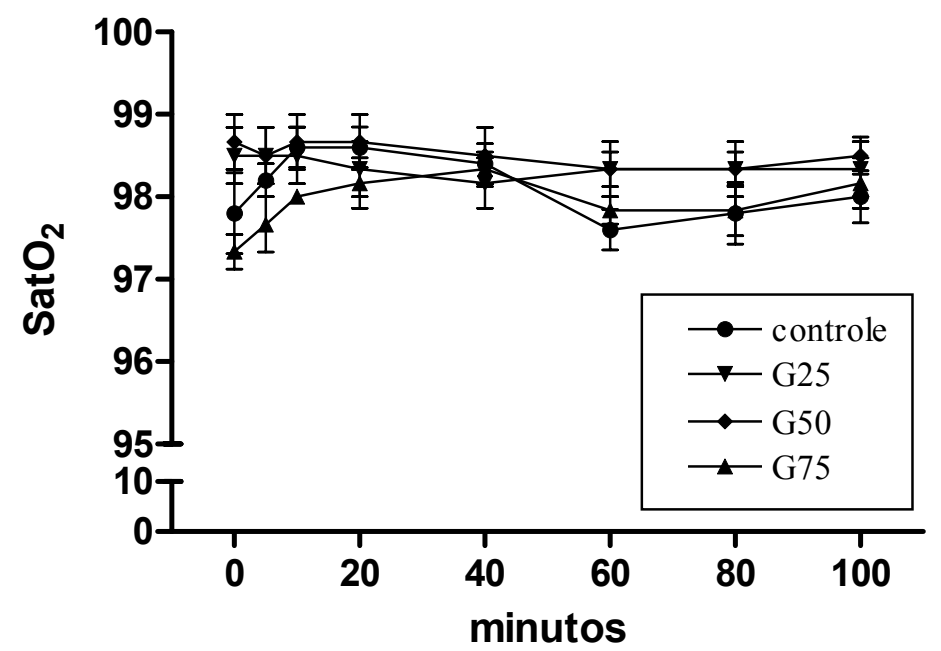

Figura 4. Saturações arteriais de oxihemoglobina $\left(\mathrm{SatO}_{2}\right)$ obtidas em cães submetidos ao bloqueio da musculatura extrínseca do bulbo ocular pelo atracúrio IV. Valores expressos em média \pm erro-padrão.

\section{REFERÊNCIAS BIBLIOGRÁFICAS}

BOLDY, K.L. Current status of canine cataract surgery. Sem. Vet. Med. (Small Animal), v.3, p.62-88, 1988.

CALLEGARI-JACQUES, S.M. Bioestatística: princípios e aplicações. 1.ed. Porto Alegre: Editora Artmed, 2003. 255p.
CHENG, K.I.; TANG, C.S.; TSAI, E.M. et al. Correlation of arterial and end-tidal carbon dioxide in spontaneously breathing patients during ambulatory gynecologic laparoscopy. $J$. Form. Med. Assoc., v.98, p.814-819, 1999.

GROSENBAUGH, D.A.; MUIR III, W.W. Accuracy of noninvasive oxyhemoglobin 
saturation, end-tidal carbon dioxide concentration, and blood pressure monitoring during experimentally induced hypoxemia, hypotension, or hypertension in anesthetized dogs. Am. J. Vet. Res., v.59, p.205-212, 1998.

GUYTON, A.C.; HALL, J.E. Tratado de Fisiologia Médica. 9.ed. Rio de Janeiro: Ed. Guanabara Koogan, 1997. 1014p.

HASKINS, S.C. Monitoring the anesthetized patient. In: THURMON, J.C.; TRANQUILLI, W.J.; BENSON, G.J. (Eds.). Lumb \& Jones' veterinary anesthesia. 3.ed. Baltimore: Lea \& Febiger Book, 1996. p.409-424.

JAKHIRANI, N.K.; MUNEERUDDLN, M.; BALOCH, R. A study of the use of atracurium in minor and major surgery. J. Pak. Med. Assoc., v.2, p.41-42, 1990. (Resumo).

KHANNA, A.K.; McDONELL, W.N.; DYSON, D.H. et al. Cardiopulmonary effects of hypercapnia during controlled intermittent positive pressure ventilation in the horse. Can. J. Vet. Res., v.59, p.213-221, 1995.

Le CORRE, F.; PLAUD, B.; BENHAMOU, E. et al. Visual estimation of onset time at the orbicularis oculi after five muscle relaxants: application to clinical monitoring of tracheal intubation. Anesth. Analg., v.5, p.305-310, 1999.

LEE, D.D.; MEYER, R.E.; SULLIVAN, T.C. et al. Respiratory depressant and skeletal muscle relaxant effects of low-dose pancuronium bromide in spontaneously breathing, isofluraneanesthetized dogs. Vet. Anest., v.27, p.473-479, 1998.

LUNA, S.P.L. Equilíbrio ácido-básico. In: FANTONI, D.; CORTOPASSI, S. Anestesia em cães e gatos. 1.ed. São Paulo: Editora Roca, 2001. p.120-129.

MAGI, E.; MULTARI, G; RECINE, C. et al. Difference between arterial and end-tidal carbon dioxide tension during surgery of lumbar herniated disk in general anesthesia. Minerva Anesth., v.60, p.381-386, 1994. (Resumo).

MARKEWITZ, B.A.; ELSTAD, M.R. Succinylcholine-induced hyperkalemia following prolonged pharmacologic neuromuscular blockade. Chest., v.1, p.248-250, 1997.
McMANUS, M.C. Neuromuscular blockers in surgery and intensive care, Part 2. Am. J. Health Syst. Pharm., v.24, p.2381-2395, 2001.

MOON, R.E. Respiratory monitoring. In: MILLER, R.D. Anesthesia. 4.ed. New York: Churchill Livingstone, 1990. p. 1129-1163.

MUIR, W.W.; HUBBELL, J.A.E.; SKARDA, R.T. et al. Fármacos bloqueadores neuromusculares. In : Manual de anestesia veterinária. 3.ed. Porto Alegre: Art Med Editora, 2001. p. 149-157.

NAGUJB, M.; MAGBOUL, M.M. Adverse effects of neuromuscular blockers and their antagonists. Middle East J. Anesthesiol., v.5, p.34l-373, 1998. (Resumo).

NUNES, N.; LAUS, J.L. Técnicas anestésicas destinadas à cirurgia ocular no cão. Braz. J. Vet. Res. Anim. Sci., v.32, p.177-180, 1995.

OSTERGAARD, D.; ENGBAEK, J.; VIBYMOGENSEN, J. Adverse reactions and interactions of the neuromuscular blocking drugs. Med. Toxicol. Adverse Drug Exp., v.5, p.351-368, 1989.

RANG, H.P.; DALE, M.M.; RITTER, J.M. Farmacologia. 4.ed. Rio de Janeiro: Guanabara Koogan, 2001. 703p.

REECE, W.O. Respiração em mamíferos. In: SWENSON, M.J. Dukes - Fisiologia dos animais domésticos. 8.ed, Rio de Janeiro: Guanabara Koogan, p.199-222, 1988.

SPRUELL, J.S.A.; CHAWLA, H.B.; CRISPIN, S.M. Routine lens extraction for the treatment of cataract in the dog. J. Small Anim. Pract., v.21, p.535-554, 1980.

TEIXEIRA-NETO, F.J.; CARREGARO, A.B.; MANNARINO, R. et al. Comparison of sidestream capnograph and a mainstream capnograph in mechanically ventilted dogs. $J$. Am. Vet. Med. Assoc., v.221, p.1582-1585, 2002.

VIZI, E.S.; LENDVAI, B. Side effects of nondepolarizing muscle relaxants: Relationship to their antinicotinic and antimuscarinic actions. Pharmacol. Ther., v.73, p.75-89, 1997. 